\section{The clinical role of the gut microbiome and fecal microbiota transplantation in allogeneic stem cell transplantation}

\author{
Israel Henig, ${ }^{1}$ Dana Yehudai-Ofir ${ }^{1,2}$ and Tsila Zuckerman ${ }^{1,2}$ \\ ${ }^{1}$ Department of Hematology and Bone Marrow Transplantation, Rambam Health Care \\ Campus and ${ }^{2}$ The Ruth and Bruce Rappaport Faculty of Medicine, Technion - Israel \\ Institute of Technology, Haifa, Israel
}

\section{ABSTRACT}

O utcomes of allogeneic hematopoietic stem cell transplantation (alloHSCT) have improved in the recent decade; however, infections and graft-versus-host disease remain two leading complications significantly contributing to early transplant-related mortality. In past years, the human intestinal microbial composition (microbiota) has been found to be associated with various disease states, including cancer, response to cancer immunotherapy and to modulate the gut innate and adaptive immune response. In the setting of allo-HSCT, the intestinal microbiota diversity and composition appear to have an impact on infection risk, mortality and overall survival. Microbial metabolites have been shown to contribute to the health and integrity of intestinal epithelial cells during inflammation, thus mitigating graft-versus-host disease in animal models. While the cause-andeffect relationship between the intestinal microbiota and transplant-associated complications has not yet been fully elucidated, the above findings have already resulted in the implementation of various interventions aiming to restore the intestinal microbiota diversity and composition. Among others, these interventions include the administration of fecal microbiota transplantation. The present review, based on published data, is intended to define the role of the latter approach in the setting of allo-HSCT.

\section{Introduction}

The past decades have witnessed important advances in the outcome of allogeneic hematopoietic stem cell transplantation (allo-HSCT), ${ }^{1}$ mainly attributed to the reduction in non-relapse mortality. ${ }^{2}$ Yet, the need for further improvement is compelling. Acute graft-versus-host disease (aGvHD) and infections are two of the main causes of early transplant-related mortality (TRM), jointly accounting for $36 \%$ and $43 \%$ of deaths by day 100 in matched related and matched unrelated transplants, respectively. ${ }^{1}$

One of the emerging and extensively explored allo-HSCT-associated issues is the change in the gut microbial flora, as well as its effect on the pathogenesis of transplant-related complications and association with transplant outcomes.

The human body hosts a hundred trillion microbial organisms; the majority of them are bacteria, predominantly colonizing the gut, with the lower intestine being most densely colonized $\left(10^{11}-10^{12}\right.$ organisms/g of intestinal content). ${ }^{3}$ The composition of bacteria in the gut is referred to as the intestinal microbiota and their collective genome is termed the "intestinal microbiome". ${ }^{3}$ The two main phyla constituting more than $90 \%$ of the gut microbiota are the Firmicutes and Bacteroidetes and among less dominant phyla are Proteobacteria, Actinobacteria, and Verrucomicrobia. ${ }^{4}$ This composition is relatively flexible and can rapidly change in response to different environmental factors, adjusting the metabolic and immunologic performance accordingly. ${ }^{5}$ Intestinal microbiota has been recently found to have a significant impact on both health and disease states. It appears to be crucial for the maturation and education of the immune system and has a role in intestinal cell proliferation, intestine vascularization and endocrine functions. Moreover, it produces energy, synthesizes vitamins, metabolizes bile acids and even inactivates drugs. ${ }^{6.13}$ The microbiome has been reported to be associated with a variety of disorders such as

\author{
Haematologica 2021 \\ Volume 106(4):933-946
}

(1)

\section{Correspondence:}

TSILA ZUCKERMAN

t_zuckerman@rambam.health.gov.il

Received: May 23, 2020.

Accepted: August 28, 2020.

Pre-published: November 26, 2020.

https://doi.org/10.3324/haematol.2020.247395

(C)2021 Ferrata Storti Foundation

Material published in Haematologica is covered by copyright. All rights are reserved to the Ferrata Storti Foundation. Use of published material is allowed under the following terms and conditions:

https://creativecommons.org/licenses/by-nc/4.0/legalcode. Copies of published material are allowed for personal or internal use. Sharing published material for non-commercial purposes is subject to the following conditions: https://creativecommons.org/licenses/by-nc/4.0/legalcode, sect. 3. Reproducing and sharing published material for commercial purposes is not allowed without permission in writing from the publisher. 
obesity, type 2 diabetes, inflammatory bowel disease, rheumatoid arthritis and multiple sclerosis. ${ }^{14-17}$ This association is also suggested to be true for cancer ${ }^{18}$ and response to cancer immunotherapy. ${ }^{19}$ The gut microbiota has a close and reciprocal relationship with the host immune system. Intestinal epithelial cells, goblet and paneth cells produce the luminal protective mucosal layer and antimicrobial peptides, allowing the transcellular transport of immunoglobulin A (IgA) antibodies. These functions regulate luminal microbial colonization. ${ }^{20}$

Homeostasis of the immune response in the gut mucosa is maintained by the balance between pro-inflammatory cells, which include T-helper 1 (Th1) cells producing interferon $\gamma$ (IFN $\gamma$ ), Th17 cells producing IL-17A and IL-22, diverse innate lymphoid cells with cytokine effector features resembling those of Th2 and Th17 cells, the antiinflammatory Foxp3+ regulatory T-cells (Tregs) and IgAsecreting B-cells. This homeostasis can be modulated by the gut microbiota. ${ }^{21-23}$ In pre-clinical studies, intestinal microbiota has been shown to regulate the expression of pro-inflammatory cytokines, human leukocyte antigen (HLA) type I and type II molecules and increase T-cell proliferation. ${ }^{18}$ Effects of the microbiota on cytokine expression and immune cell subsets are not limited to the gut, and are extended to regional mesenteric and systemic lymph nodes. ${ }^{24}$ Furthermore, while some bacterial strains can induce pro-inflammatory intestinal Th17 cells, ${ }^{25}$ others induce anti-inflammatory Tregs ${ }^{26,27}$ and can thus ameliorate inflammatory colitis. ${ }^{28}$ Moreover, human host gut microbiota has been shown to correlate with expression pattern of the cytokines secreted from peripheral blood mononuclear cells isolated from the host. ${ }^{29}$ Microbial metabolites such as the short chain fatty acid (SCFA) butyrate or indole derivatives produced by tryptophan metabolism act to maintain the intestinal epithelial cell health, mucosal barrier, and to promote anti-inflammatory responses. ${ }^{30,31}$

Currently available molecular techniques allowing rapid and wide genomic sequencing enable extensive exploration of the microbiome. The most commonly used method is the $16 \mathrm{~S}$ ribosomal RNA sequencing by PCR. Bioinformatics analysis tools assign the sequences to microbial taxon at different taxonomic levels. Other methods include shotgun next-generation metagenomics sequencing enabling massive and deeper genomic sequencing and allowing better identification of taxonomic species and potential functional pathways of the organisms, metatranscriptomics using high throughput RNA sequencing to profile gene expression, metaproteomics capable to provide large-scale characterization of the entire proteins in the environmental sample and metabolomics, identifying and quantifying all metabolites in the tested samples. ${ }^{32,33}$ The two main microbiome features that have been widely characterized in health and disease are its diversity and the abundance of specific bacteria or bacterial subgroups. ${ }^{34}$

The revelation of significant relationship between the microbiome, the immune system and disease has led to interventional studies aiming to normalize the microbiome composition and diversity thus ameliorating disease conditions. One of such interventions is the use of fecal microbiota transplantation (FMT), the term referring to the transfer of the fecal microbial content from a healthy individual into the intestine of a diseased individual. FMT, the standard of care for refractory or recurrent Clostridium difficile infection (CDI), proved to be highly effective in this condition. At the same time, mixed results were demonstrated in the studies evaluating the use of FMT for the management of inflammatory bowel disease, irritable bowel syndrome and hepatic encephalopathy. To date, FMT application for indications other than CDI has been limited to the experimental setting only. ${ }^{35,36}$

The setting of allo-HSCT imposes a significant disruption on the gut microbiome homeostasis through a variety of mechanisms (all part of the transplantation procedure), such as the use of broad-spectrum antibiotics, dietary changes (restriction), gut epithelial damage by conditioning regimens and introduction of a donor immune system.

Data from clinical studies support the association of alterations in the gut microbiome profile, mainly loss of diversity and change in composition during allo-HSCT, with patient outcomes such as aGvHD, GvHD-related mortality, non-relapse mortality (NRM) and overall survival (OS). ${ }^{37-40}$ Moreover, the gut microbial composition is reported to have an impact on infection risk, including $\mathrm{CDI}$ and blood stream infections (BSI), in this clinical setting. ${ }^{38,41}$ Findings of these associations have led to a preponderance of research in this field, ${ }^{42}$ and although the causeand-effect relationship between the microbiome and transplant complications has not been unequivocally established, many ongoing clinical trials are implementing various interventions aiming to maintain microbiome diversity, thus potentially preventing transplant-related complications and treating a GvHD. These interventions include the use of probiotics, ${ }^{43}$ prebiotics, ${ }^{44}$ change in antibiotic prophylaxis $^{45}$ and administration of FMT. $^{46}$ This review appraises the currently available evidence on the association of gut microbiota and allo-HSCT and analyzes a potential role of FMT in allo-HSCT, by presenting two illustrative clinical cases, where effects on the gut microbiota composition could be employed either as a prophylactic or therapeutic measure.

\section{Case 1}

A 54-year old male, with mutated FLT3-ITD acute myeloid leukemia (AML) in complete remission (CR) after induction and re-induction chemotherapies, during which he acquired gut colonization with carbapenemresistant Klebsiella pneumoniae. He underwent an alloHSCT from a mismatched 9/10 unrelated female donor with myeloablative conditioning (busulfan, fludarabine) and received levofloxacin for infection prophylaxis. During the transplantation period, he had a BSI event with extended spectrum $\beta$ lactamase Escherichia coli (E. coli) treated with meropenem for 10 days, followed by a CDI event treated with oral vancomycin. His neutrophils engrafted on day +15 and on day +33 he developed diarrhea and was diagnosed with grade 3 acute lower gastrointestinal (GI) GvHD that was steroid refractory.

This case raises a number of important questions related to the role of gut flora in allo-HSCT.

\section{Is the microbiome already disrupted prior to allogeneic hematopoietic stem cell transplantation conditioning?}

There is ample evidence suggesting that the pre-transplant patient microbiome is already disrupted. The insult to the microbiome starts with preceding chemotherapy and 
antibiotic exposure. Galloway-Pena et al. ${ }^{47}$ analyzed 487 stool samples from 30 AML patients and found that their pre-induction microbiome diversity was not significantly different from that of healthy volunteers participating in the Human Microbiome Project (HMP). However, following neutrophil recovery, patient microbiome composition changed, with a significant decrease in diversity. Importantly, this reduction in diversity was associated with an increased risk of infections. The use of carbapenem antibiotics for more than 3 days during induction elevated the risk for a subsequent loss of diversity. ${ }^{47}$ Moreover, exposure to anti-anaerobic antibiotics, like piperacillin-tazobactam, ticarcillin, meropenem, clindamycin and metronidazole, within the 3 months preceding allo-HSCT was associated with a significant decrease in pre-transplant microbiome diversity. ${ }^{38}$ With more courses of intensive chemotherapy, such as re-induction or salvage, the microbiome disruption was shown to enhance, leading to ecosystem instability and outgrowth of pathogenic bacteria like Enterococcus. ${ }^{48}$ This disruption in patient microbiome continued up to the time of allo-HSCT, as shown in the largest to date inter-center effort, where 8,767 sequential stool samples were collected from 1,362 patients prior to and throughout the transplantation period and analyzed using $16 \mathrm{~S}$ ribosomal RNA sequencing. The pre-transplant microbiome of patients obtained on days -30 to $-6(n=606)$, was compared to that of healthy volunteers $(n=246)$, demonstrating a significant reduction in diversity in patient microbiome..$^{37}$ Additionally, evidence from another recently published study showed that the pre-transplant microbiome and the one derived from healthy controls differed in composition, displaying decreased abundance of beneficial bacteria of genera Bifidobacterium and butyrate producing genera such as Faecalibacterium and Lachnospiraceae in the former case. ${ }^{49}$ To conclude, pre-transplant microbiome disruption is clearly evident.

\section{What is the microbiome status during the transplantation period and at time of recovery?}

Data from several studies demonstrate that during the transplantation course, the microbiome diversity significantly decreases and its composition changes. ${ }^{37,50}$ The lower-diversity microbiome is reported to be characterized by abundance of pathogenic bacteria such as Enterococcus, Klebsiella, Escherichia, Staphylococcus and Streptococcus. The single taxonomic unit domination (abundance $\geq 30 \%$ ) peaks at 1 week post-transplant, which is followed by a subsequent moderate decrease. The most common dominating taxonomic groups belong to the genera Enterococcus and Streptococcus. ${ }^{37}$ Along the same lines, other studies have found the Enterococcus genus to be more prolific during the first month posttransplant, with significantly higher abundance in patients with active or subsequent aGvHD. ${ }^{51,52}$ Following allo-HSCT, the microbiome recovery appears to be prolonged and incomplete. In a large cohort of patients ( $n=753$ ), the post-transplant recovery of the gut microbiota has been reported to start around day +50 , but even by day +100 the composition and bacterial abundance observed pre-transplant have not been fully achieved..$^{53}$ Moreover, in some patients, microbiota has remained disrupted even 1 year after HSCT, this being particularly the case with butyrate-producing bacteria and Bifidobacterium. ${ }^{54}$ Eventually, the effect of environmental insult on the intestinal microbiota during allo-HSCT can be so severe that its recovery may require a long time.

\section{Is the disrupted microbiome in allogeneic hematopoietic stem cell transplantation recipients clinically significant?}

In the above-mentioned study by Peled et al., reduced microbiome diversity both pre-transplant (days -30 to -6) and peri-engraftment (days +7 to 21 ), was shown to be significantly associated with lower 2-year OS, while a persistent decrease of this parameter in the latter period was also associated with higher 2-year treatment-related mortality (TRM). Moreover, lower peri-engraftment microbiome diversity in T-cell replete allo-HSCT corresponded to increased GvHD-related mortality, which was not observed in T-cell depleted transplantations. This difference suggests a connection between the microbiota and T-cell alloreactivity. ${ }^{37}$ Liu et al. revealed a similar association of pre-transplant diversity with mortality as well as a correlation between post-transplant microbiome disruption and acute GI GvHD risk. ${ }^{55}$ Furthermore, in a study of 66 patients whose stool specimens were analyzed weekly during the transplantation period up to day +100 , Golob et al. found a trend of association between near-engraftment low microbiome diversity and the risk for grade 3-4 aGvHD. ${ }^{56}$ Likewise, Mancini et al. evaluating a cohort of 100 patients, observed a significant connection between low microbiome diversity by day +10 and an increased risk for early (within 30 days) aGvHD. ${ }^{38}$

A number of studies also reported an impact of pre- or post-transplant bacterial abundance on patient outcomes (Table 1). Results of a two-cohort study (a total of 115 adult patients) conducted at the Memorial Sloan Kettering Cancer Center (MSKCC) demonstrated that increased abundance of the genus Blautia, including anaerobic commensal bacteria, observed 12 days post-transplant, was associated with reduced GvHD-related mortality and improved OS. At the same time, the use of antibiotics with anti-anaerobic activity and total parenteral nutrition (TPN) correlated with loss of Blautia. ${ }^{57}$ In the pediatric setting, Biagi et al. reported an association of pre-transplant high abundance of Blautia and low abundance of Fusobacterium with diminished risk for grade 2-4 acute GI GvHD. ${ }^{58}$ Additionally, pre-transplant Enterobacteriaceae abundance of $>5 \%$ was associated with an increased risk of BSI and Lachnospiraceae abundance of $\leq 10 \%$ appeared to correspond to increased mortality. ${ }^{38}$ In a large study from the MSKCC, very high abundance of a bacterial group, mainly composed of Eubacterium limosum, in pretransplant samples or the presence of this group in periengraftment samples was found to correspond to a decreased relapse risk, ${ }^{59}$ once again emphasizing the association of the microbiome and T-cell immunity. Furthermore, in the study from the Osaka University, ${ }^{54}$ Enterococcus relative abundance of $\geq 1 \%$ at 1 month posttransplant appeared to be indicative of poor OS, with a 2year survival of $83.9 \%$ for patients with relative abundance of Enterococcus $<1 \%$ versus $47.6 \%$ for those in whom this parameter was $\geq 1 \%$. It is noteworthy that none of the surviving patients at 1 year post-transplant displayed Enterococcus abundance higher than 1\%, sug- 
Table 1. Intestine microbial changes in diversity and abundance during pre-transplant and peri-engraftment periods, associated with outcomes of allogeneic hematopoietic stem cell transplantation

\begin{tabular}{|c|c|c|c|c|}
\hline Outcome & Pre-transplant & Ref. \# & Peri-engraftment & Ref.\# \\
\hline Overall survival $\downarrow$ & Diversity $\downarrow$ & $37 ; 55$ & $\begin{array}{l}\text { Diversity } \downarrow \\
\text { Blautia (day }+12) \downarrow \\
\frac{\text { Enterococcus } R A}{(\text { day }+30)} \geq 1 \%\end{array}$ & $\begin{array}{l}37 \\
57 \\
54\end{array}$ \\
\hline $\begin{array}{l}\text { Transplant- related } \\
\text { mortality } \uparrow\end{array}$ & $\begin{array}{l}\text { Lachnospiraceae } \\
\leq 10 \%\end{array}$ & 38 & $\begin{array}{l}\text { Peri-engraftment diversity } \downarrow \\
\text { Engraftment diversity } \downarrow\end{array}$ & $\begin{array}{l}37 \\
40\end{array}$ \\
\hline $\begin{array}{l}\text { Acute gastrointestinal } \\
\text { GvHD risk } \uparrow\end{array}$ & $\begin{array}{l}* \underline{\text { Blautia }} \downarrow \\
\neq \text { Diversity } \downarrow \\
* \underline{\text { Fusobacterium }} \uparrow\end{array}$ & $\begin{array}{l}58 \\
56 \\
58\end{array}$ & 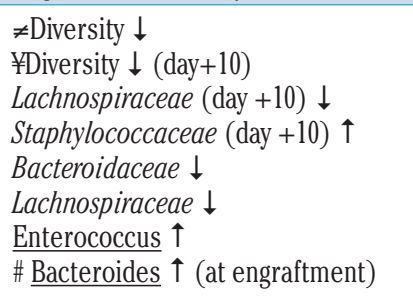 & $\begin{array}{l}56 \\
38 \\
38 \\
38 \\
56 \\
56 \\
52 \\
58\end{array}$ \\
\hline GvHD-related mortality $\uparrow$ & & & $\begin{array}{l}\text { I Diversity } \downarrow \\
\text { Blautia (on day }+12) \downarrow\end{array}$ & $\begin{array}{l}37 \\
57\end{array}$ \\
\hline Blood stream infections $\uparrow$ & $\begin{array}{l}\text { Enterobacteriaceae } \\
(\mathrm{RA}>5 \%)\end{array}$ & 38 & $\begin{array}{l}\text { Enterococcus }(\mathrm{RA} \geq 30 \%)->\mathrm{VRE} \uparrow \\
\text { Proteobacteria }(\mathrm{RA} \geq 30 \%)->\mathrm{GN} \uparrow\end{array}$ & $\begin{array}{l}50 \\
50\end{array}$ \\
\hline Relapse $\downarrow$ & Eubacterium limosum $\uparrow \uparrow$ & 59 & Eubacterium limosum presence & 59 \\
\hline
\end{tabular}

$\downarrow$ represents a decrease in risk; $\uparrow$ represents an increase in risk; $\downarrow$ next to diversity means loss of diversity; $\downarrow$ or $\uparrow$ next to a bacterial taxa represent decrease or increase in relative abundance, respectively. Different bacterial taxonomic rank is marked as follows: phyla (bold and italics), family (italics), genus (underlined) and species (bold). * taxa associated with grade $2-4$ acute gastrointestinal graft-versus-host disease (GvHD). $\neq$ diversity associated with grade 3-4 acute GvHD (aGvHD). ¥ diversity associated with early aGvHD, by day $30 . \#$ a trend $(P=0.05)$. ITT-cell replete transplants. RA: relative abundance;VRE: vancomycin-resistant Enterococcus; GN: gram negative.

gesting that this cutoff could serve as a prognosticator of a long-term outcome in this clinical setting. ${ }^{54}$ The above evidence suggests that the microbiota changes before and during allo-HSCT are significantly associated with transplant complications and outcomes and might even serve as a predictive marker in this setting.

\section{Can prophylactic fecal microbiota transplanta- tion reduce the risk of infections during allo- geneic hematopoietic stem cell transplantation?}

In allo-HSCT recipients, curtailment of infection risk is crucial for reducing TRM, particularly due to increased frequency of BSI with multidrug resistant (MDR) bacteria. MDR colonization is established to range between $16 \%$ for gram-negative bacteria and 39\% for vancomycin-resistant Enterococcus (VRE). While BSI have been reported in $16-41 \%$ of patients colonized with MDR bacteria, findings regarding a possible association of such colonization with TRM or infection-related mortality are inconclusive. ${ }^{60-62}$ In addition, MDR gram-negative colonization has neither been found to correspond to an increased risk for sepsis. ${ }^{38,63}$ In the lack of clear evidence, proof-of-concept studies are becoming of increasing importance. Battipaglia et al. ${ }^{64}$ have evaluated four patients colonized with MDR bacteria who had received FMT on days -46 to -9 before transplant with an aim to limit the risk for infectious complications during HSCT. All the four patients responded with decolonization of the MDR bacteria. One patient developed grade 3 acute gut GvHD on day +30 after transplant (day +51 after FMT) and two others developed bacteremia with sensitive bacteria. Notably, despite receiving broad-spectrum antibiotics during the transplantation period, none of the patients had recolonization of the gut with MDR bacte- ria. ${ }^{64}$ Similar results were reported in a 63 -year old HSCT recipient. $^{65}$

The ongoing ODYSSEE trial (clinicaltrials gov. Identifier: 02928523) is aimed at reducing complications that may arise as a result of a loss of microbiota diversity, including infectious complications, poor nutritional status, prolonged hospitalization, as well as therapy discontinuation due to induction treatment-related toxicity in AML patients. Twenty newly diagnosed patients collected pre-induction autologous stools. This autologous FMT was later administered as enema after neutrophil recovery and prior to consolidation chemotherapy. Preliminary results demonstrated safety of this approach, with evidence of stool diversity restoration 10 days after FMT and reduction in antibiotic resistant gene copy count by $43 \%$. Yet, clinical efficacy of this method still needs to be confirmed. ${ }^{66}$

An important pathogen to consider for intervention with FMT is Clostridium difficile. The incidence of CDI during allo-HSCT varies between $13 \%$ and $30 \%$, mostly in the first month after transplant. ${ }^{67-69}$ The disease is usually of mild-to-moderate severity, with good response to treatment; there is no association with TRM, and its possible correlation to subsequent acute GI GvHD is indefinite. ${ }^{68-70}$ Given these facts, and the paucity of data on potential efficacy of prophylactic FMT in reducing the risk of CDI among Clostridium difficile carriers, FMT prophylaxis may not be required for this indication.

As for the treatment of recurrent CDI, results of three small studies demonstrate safety of FMT administration to a total of 16 patients with recurrent CDI after alloHSCT, with only three patients recurring after the procedure. .1173 $^{-71}$

Currently available data are insufficient to definitively conclude that prophylactic FMT will reduce the infection rate in the allo-HSCT setting. 


\section{Can prophylactic fecal microbiota transplantation reduce the risk of acute graft-versus-host disease or transplant-related mortality?}

The incidence of clinically significant aGvHD ranges between $22 \%$ in allo-HSCT from a matched related donor to $29 \%$ in case of a mismatched unrelated donor, with grade 3-4 disease incidence being $8.6 \%$ and $12 \%$, respectively. ${ }^{24}$ Whether any intervention that restores the microbiome composition could also decrease aGvHD rates is yet to be revealed. Hitherto, only two small studies have reported results of using prophylactic FMT in the post-engraftment period. In the study by Defillip et al. ${ }^{25}$ aiming to evaluate safety and feasibility of early restoration of the gut microbiome, frozen capsules of FMT derived from unrelated donors were administered to 13 allo-HSCT recipients 4 weeks after neutrophil engraftment. No FMT-related bacteremia events occurred and two cases of acute GI GvHD were registered. Analysis of stool composition indicated improvement in intestinal microbiome diversity after FMT that was mainly attributed to operational taxonomic units (OTU) originating from the FMT donor. ${ }^{25}$ In the study by Taur et al..$^{53}$ within 3-28 days of engraftment, patients not receiving broadspectrum antibiotics, not critically ill and with low abundance of Bacteroides ( $<0.1 \%$ of the total $16 \mathrm{~S}$ sequencing) at that time period, were randomized to either receive autologous FMT $(n=14)$ or to a control group $(n=11)$.

Solely the FMT group was found to reconstitute their microbiome diversity and composition to the pre-transplant state. Of note, the use of autologous FMT raises concern for disrupted microbiota due to prior antibiotic exposure. $^{53}$

These data suggest feasibility and safety of prophylactic FMT; however, its clinical benefit has not been demonstrated yet.

\section{Should additional interventions along with fecal microbiota transplantation aiming to attenuate mircobiome disruption be considered?}

Given that a variety of factors could affect the microbiome diversity and composition during the transplantation course, their adequate control might potentially preclude such microbiome changes. The question remains whether FMT alone is sufficient enough or it should be combined with other interventions to provide the required control.

\section{Transplant conditioning}

Conditioning chemotherapy itself has a disruptive effect on the microbiome, as found by Montassier et al. ${ }^{26}$ who evaluated eight lymphoma patients undergoing autologous HSCT with the BEAM (carmustine, etoposide, cytarabine arabine, melphalan) protocol. Since none of the patients received nasogastric tube nutrition, total parenteral nutrition, ciprofloxacin prophylaxis or systemic antibiotic treatment, only the chemotherapy effect on the microbiome was measured. Compared to pretransplant samples, those drawn at 1 week post-conditioning demonstrated significantly reduced diversity, decreased abundance of Firmicutes and Actinobacteria and increased presence in bacteroides and proteobacteria, indicating chemotherapy-induced disruption of the intestinal microbiota. ${ }^{26}$ Of note, this disruptive effect might be related to etoposide, which has bacterial inhibitory activity. ${ }^{27,28}$ Remarkably, the post-transplant decrease in microbiome diversity appeared to be more profound when more intensive conditioning was applied. ${ }^{74}$ However, reducing the conditioning intensity was not shown to consistently decrease the rate of aGvHD. ${ }^{75}$ Moreover, it might increase the relapse rate and decrease long-term OS. ${ }^{76,77}$ Therefore, changing the conditioning regimen in an attempt to attenuate the insult on the microbiome is not currently recommended.

\section{Diet}

Dietary interventions such as TPN, prebiotics and probiotics could potentially influence the microbiome composition before or during the transplantation course. TPN administration was reported to be associated with decreased recovery of post-transplant (up to day +120 ) diversity compared to enteral nutrition. In addition, SCFA levels in the gut content were found to be lower in the TPN group..$^{78}$ Iyama et al. retrospectively compared a group of patients whose diet was supplemented with prebiotics, i.e., glutamine, fiber and oligosaccharides (GFO) with a group that did not receive such supplementation. GFO was started 7 days before conditioning and continued up to day +28 . In the GFO group, duration of diarrhea, mucositis and TPN requirement was shorter and the weight loss was also less prominent. ${ }^{44}$ An ongoing prospective trial (clinicaltrials gov. Identifier: 02763033) is evaluating the efficacy of resistant potato starch supplementation between day -7 and day +100 in HSCT recipients. This starch is a non-absorbable carbohydrate that is metabolized by the anaerobic commensal bacteria to produce the SCFA butyrate, ${ }^{79}$ shown to reduce the severity of acute GI GvHD in an experimental model. ${ }^{31}$ Preliminary results demonstrate the feasibility of this approach in terms of patient compliance, increase in intestinal butyrate levels and abundance of butyrate producing bacteria. ${ }^{80}$ As for probiotic supplementation, the available data do not suggest its influence on the microbiome composition or clinical outcomes. It is worth mentioning that the products used in the studies contained only one bacterial strain and not a diversity of bacteria, ${ }^{43,81}$ and safety of probiotic administration is of concern in immunocompromised patients. ${ }^{82}$

The loss of diversity during the transplantation course is accompanied with microbiome domination by single taxonomic units such as Enterococcus. ${ }^{37}$ This enterococcal expansion has been found to be most prominent in patients developing acute GI GvHD. ${ }^{52}$ Stein-Thoeringer et al. have shown in a gnotobiotic mouse model of alloHSCT that enterococcal expansion in the gut depends on lactose and its depletion decreases the enterococcal abundance and thus attenuates GvHD severity. Furthermore, in patients with a lactose malabsorption genotype, Enterococcus abundance appears to be higher than in patients without this genotype. ${ }^{83}$ This finding may give rise to a new approach to dietary intervention during HSCT. Interestingly, in the study by Khandelwal et al., where pediatric allo-HSCT patients under the age of 5 were treated with ready to eat human milk and breast feeding $(n=24)$ or formula $(n=14)$, plasma levels of IL6, IL10, and Reg3 $\alpha$ were significantly lower in the group receiving human milk. The microbiome composition also 
differed between the two groups, with an increase in pathogenic species such as $E$. coli in the formula-receiving group. Despite the fact that human milk oligosaccharides are metabolized to SCFA by the commensal bacteria, butyrate levels in the stool were similar in both groups. Moreover, no significant difference in the rate of grade 24 acute GI GvHD between the groups was revealed. However, the limited size of this study calls for cautious interpretation of these encouraging results. ${ }^{84}$ Overall, dietary interventions emerge as a promising way to shape the intestinal microbiota during allo-HSCT. However, results are too preliminary and more research is required before implementing any of these methods.

\section{Antibiotic treatment}

The antibiotic treatment applied during the transplantation course is the main factor affecting the microbiome. Quinolone prophylaxis during afebrile neutropenia and systemic broad-spectrum antibiotic treatment with piperacillin-tazobactam or meropenem are widely accepted. ${ }^{85-87}$ However, data demonstrate that the use of other antibiotics can better preserve gut beneficial commensals and is associated with improved outcomes.

The study from the University of Regensburg in Germany employed the non-absorbable antibiotic rifaximin and compared it to ciprofloxacin and metronidazole used in a historic cohort of patients for infection prophylaxis during allo-HSCT. ${ }^{45}$ Antibiotics were given from day -8 up to engraftment. The urine 3-indoxyl sulfate (3-IS) level was measured as a marker of microbiome diversity. ${ }^{88}$ In the rifaximin cohort, the pre-engraftment 3-IS levels were significantly higher without an increase in the sepsis rate or colonization with pathogenic bacteria. This group had significantly lower TRM, prolonged OS and the acute GI $\mathrm{GvHD}$ rate tended to be lower in these patients. The observed advantage remained evident even in patients who later received systemic antibiotics for neutropenic fever. ${ }^{45}$

Given the major role of microbiome diversity preservation during allo-HSCT and an association of impaired diversity with acute GI GvHD and adverse patient outcome, Weber et al. further compared the effects of various prophylactic and systemic antibiotics in an attempt to identify the ones that could spare commensal bacteria. ${ }^{89}$ At 10 days post-transplant, the patient groups receiving rifaximin without systemic antibiotics or rifaximin with systemic antibiotics maintained their microbiome diversity and Clostridia abundance and had higher 3-IS levels compared to patients treated with ciprofloxacin/metronidazole \pm systemic antibiotics. These results suggest that rifaximin could better preserve microbiome diversity even when systemic broad-spectrum antibiotics are administered during transplantation. Moreover, in the study conducted in two Canadian hospitals and assessing the effect of antibiotic prophylaxis or treatment given before day 0 on frequency of aGvHD and mortality, the authors compared the outcome of a cohort of patients exposed to antibiotics $(n=239)$ to those who did not receive this therapy $(n=261) .{ }^{90}$ The antibiotic-receiving group demonstrated a significantly higher incidence of grade 2-4 aGvHD and significantly shorter $O S$ at 1,2 and 10 years posttransplant, indicating an association between the deleterious effect of such treatment on intestinal bacteria and inferior patient outcome.

Importantly, early start of systemic antibiotics (before engraftment) was found to be associated with a lower 3-
IS urine level and decreased Clostridia abundance in the stool. Furthermore, the TRM rate in such cases was higher than in patients who did not require systemic antibiotics during HSCT or started them after engraftment. ${ }^{91}$

Similarly, systemic treatment with piperacillin-tazobactam and meropenem was reported to correlate with decreased microbiome diversity during the transplanta$\operatorname{tion}^{37}$ and significant loss of commensal anaerobic bacteria. ${ }^{92}$ In pediatric patients, Simms-Waldrip et al. ${ }^{93}$ found that higher load of anti-anaerobic antibiotics was associated with a significant decrease in anti-inflammatory Clostridia (AIC) abundance, and in patients with aGvHD the abundance decrease was severe (10-log fold) compared to patients without GvHD. In a mouse allo-HSCT model, clindamycin administration was associated with AIC decrease and more severe GvHD, while re-administration of AIC increased its levels in the gut and improved survival. ${ }^{93}$ Additionally, Lee et al..$^{94}$ compared patients who did not require any systemic antibiotic treatment during the transplantation course with those who received cefepime and those who were treated with carbapenem antibiotics. The carbapenem group displayed a significant loss of microbial diversity at engraftment and an increased rate of acute GI GvHD (32.1\%) compared to the noantibiotics group (11.6\%). Interestingly, the cefepime group retained a diverse microbiome, demonstrating only a trend to a higher GI GvHD rate $(26.4 \%)$.

Furthermore, a large multicenter study retrospectively evaluating 857 patients revealed that the use of piperacillin-tazobactam and imipenem-cilastatin was associated with increased 5-year GvHD-related mortality ${ }^{95}$ while this was not observed in patients receiving cefepime and aztreonam. The former antibiotics caused a significant decrease in abundance of Bacteroidetes and Lactobacillus compared to the latter ones. These results suggest that some antibiotics may be more beneficial than others in the setting of allo-HSCT, and that this beneficial effect is related to the antibiotic ability to be less detrimental to intestinal commensal bacteria. ${ }^{95}$ Findings in the pediatric setting were consistent with these data, and exposure to anti-anaerobic antibiotics was reported to result in a significant decrease in butyrate-producing bacteria and the butyrate level in luminal content by day +14 . Pediatric patients who later developed aGvHD had a significantly lower butyrate level at that time point than patients without GvHD.96

It was also demonstrated that specific antibiotic use during allo-HSCT could change the abundance of specific taxa which was associated with BSI risk. In a cohort of 94 patients, Taur Y et al..$^{50}$ found that domination of the gut microbiome (abundance $\geq 30 \%$ ) by single bacterial taxa Enterococcus and Streptococcus occurred at the peri-engraftment period (days +10 to +20 ) in two thirds of the patients. However, treatment with metronidazole increased the risk for enterococcal domination by 3-fold, and this domination elevated the risk for VRE bacteremia by 9 -fold. Altogether, these data establish an essential role of antibiotics in disrupting or preserving the intestinal microbiota during allo-HSCT.

\section{Case 1: conclusions}

Several issues should be considered in decision-making regarding the appropriate management of this case. This 
Table 2. Clinical trials of fecal microbiota transplant in allogeneic hematopoietic stem cell transplantation.

FMI aim Study (ref.) or +NCT number $\begin{gathered}\text { Number } \\ \text { of patients }\end{gathered}$ Outcomes

\section{Prophylactic}

Reduce pathogenic bacteria colonization pre-transplantation Malard et al. ${ }^{66}$

Battipaglia et al..$^{64}$

Innes et al. . $^{65}$

Restore microbiome diversity post-transplantation Defillip et al. . $^{25}$ Taur et al. ${ }^{53}$
20 Restoration of diversity, reduction in antibiotic-resistant gene copy count All decolonized

All decolonized

$13 \quad$ Increase in diversity

Random: Increase in diversity

control 11

\section{Therapeutic}

Recurrent CDI

Steroid refractory/dependent acute GI GvHD

Webb et al. ${ }^{71}$
Moss et al..$^{72}$
Bluestone et al. ${ }^{73}$

Spindelboeck et al. ${ }^{198}$

Kakihana et al. ${ }^{46}$

Kaito et al..$^{99}$

Zhang et al. $1^{100}$

Zhong et al. ${ }^{101}$

Shouval et al. ${ }^{102}$

Malard et al. ${ }^{103}$

Qi et al. ${ }^{104}$

van Lier et al. ${ }^{105}$

'Ongoing clinical trials in GI acute GvHD

Bilinski et al. ${ }^{106}$

NCT04269850
NCT03819803
NCT03812705
NCT04285424
NCT03359980 (HERACLES trial)

7

No recurrence in 6

No recurrence in 4

$3 \quad$ No recurrence in 1

32 CR, 1 PR, 3 died

43 CR, 1 PR

$1 \quad$ CR

$1 \quad \mathrm{CR}$

$1 \quad \mathrm{CR}$

72 CR, 1 PR, 4 died

83 CR, 1 VGPR, 2 PR, 3 died

88 ORR, 2 relapsed, 4 died

$15 \quad 11 \mathrm{CR}, 5$ relapsed

105 ORR, CR 4, SD 1

$20 \quad$ Response and OS

${ }^{*} 15 \quad$ Response

$30 \quad$ Response

$30 \quad$ Response

*32 Response and OS

FMT: fecal microbiota transplant; ${ }^{*} \mathrm{NCT}$ number: clinicaltrials.gov Identifier. ${ }^{\mathrm{T}}$ Recruiting or completed from ClinicalTrials.gov; ${ }^{*} \mathrm{Estimated}$ enrollment; GI: gastrointestinal tract; GvHD: graft-versus-host disease; CR: complete remission; PR: partial remission;VGPR: very good partial remission; SD: stable disease; OS: overall survival; ORR: overall response rate.

patient has pre-transplant intestinal microbiota disruption and assumed colonization by MDR bacteria and probably by Clostridium difficile. His risk for aGvHD is high, since he has undergone allo-HSCT from a mismatched unrelated donor. Quinolone prophylaxis and meropenem treatment for BSI have further disrupted his intestinal microbiota. The existence of pre-transplant microbiota disruption, mainly attributed to the use of broad-spectrum antibiotics during intensive chemotherapy, is associated with increased TRM, shorter OS and GvHD-related mortality. Pre-transplant FMT can potentially enrich the microbiome diversity and eradicate MDR bacteria or Clostridium difficile; however, without controlling such factors as antibiotic prophylaxis and the type of systemic antibiotic therapy employed, the intervention by FMT may not completely achieve its goals.

So far, no data are available regarding a clinical benefit of prophylactic pre-transplant FMT.

While an association between peri-engraftment microbiome low diversity and patient outcome is established, implying potential feasibility of FMT use at that stage, data regarding FMT application before engraftment are not available, and for safety reasons this approach will probably not be attempted. Results of several small-scale studies suggest safety and feasibility of post-engraftment FMT in restoring microbiome diversity (Table 2); however, it remains unknown if this strategy could decrease the risk for aGvHD-related mortality and TRM.

As for dietary interventions at this period, their efficacy is still under investigation. Choosing a different antibiotic prophylaxis, such as rifaximin and systemic antibiotics such as cefepime, looks promising. Nevertheless, new strategies need to be tested to prove their non-inferiority in $\mathrm{OS}^{85}$ and to establish less disruption for the microbiome (clinicaltrials gov. Identifier: 03078010), especially since fourth-generation cephalosporins have been found in one study to be associated with an increased risk for aGvHD. ${ }^{97}$

\section{Case 1: recommendations}

In this case, based on the currently available data, we do not recommend prophylactic administration of pretransplant or post-engraftment FMT. 


\section{Case 2}

A 25-year old female with intermediate-risk AML in CR underwent an allo-HSCT with BuCy myeloablative conditioning from her matched sibling. Her neutrophils engrafted by day +14 . On day +34 she developed grade 3 aGvHD of the lower GI tract which was steroid refractory (SR). She did not respond to the addition of budesonide, extracorporeal photopheresis (ECP), mofetil mycophenolate or infliximab.

\section{Can fecal microbiota transplantation mitigate prevailing acute gastrointestinal graft-versus- host disease?}

The current data regarding the use of FMT for the treatment of acute GI GvHD are limited to case reports and small case series (Table 2). A total of 58 described patients were treated with FMT for SR GI grade $2-4 \mathrm{aGvHD}$. The FMT source was an unrelated donor in 36 cases, a related donor - in six cases and in eight cases a commercial pooled highly diverse FMT was used. FMT was processed and either given fresh within a few hours of collection or it was frozen and later thawed before administration. FMT was administered orally as packed capsules, through a nasogastric/nasoduodenal tube or an enema. Of 58 patients, 28 received FMT after two or more therapy lines, while 19 received it as second-line therapy right after steroid failure. Response was observed in $74 \%$ (43 of 58) of patients, with complete response in $57 \%$ (33 of 58) and partial response in $17 \%$ (10 of 58). Complete response was observed in $73 \%$ of patients receiving FMT as second-line therapy. Ten of the responding patients relapsed and 29 patients were alive at the last follow-up (54\%; 29 of 54 patients with available data).

Response to treatment was seen within a median of 14 days (range: 3-28), with a median of two FMT (range: 1-7), and a median of 7 days between treatments (range: 260). 4 . $989-106$

Infectious complications occurred in 11 patients. Two had sepsis with bacteria not originating from FMT, ${ }^{102}$ and one patient developed diarrhea due to Norovirus that was traced to FMT. ${ }^{106}$ Other infections were attributed to the severe immunocompromised state of patients. However, a possible association with FMT could not be ruled out. In responding patients in whom the stool microbiome was sequenced post-FMT, it was found to be significantly more diverse and enriched with Bacteroides, Lactobacillus, Bifidobacterium and Faecalibacterium compared to pre-FMT microbiome. (6,98-101 $^{4}$ Notably, the diversity increased only upon discontinuation of anti-anaerobic systemic antibiotic treatment, such as piperacillin-tazobactam. However, continuous use or re-initiating treatment with cefepime did not reduce FMT efficiency. ${ }^{46,98,99}$

These results are highly encouraging and support FMT therapy to be relatively safe and effective in SR GI aGvHD.

\section{Case 2: conclusions}

Available data suggest a potentially beneficial effect of FMT in acute lower GI GvHD. It should probably be used earlier rather than later, so that patients' response will not be overcome by infectious complications related to exten- sive immunosuppressive therapy. Discontinuation of antibiotic treatment prior to FMT administration appears to be an important factor contributing to successful response. If antibiotic treatment is required, using cefepime may allow attenuating microbiome insult while maintaining clinical response.

Current information is based on case reports and small series with a wide variability in patient selection, FMT preparation and mode of administration. However, the reported feasibility, safety and clinical benefit appear to be similar across the studies, implying that intestinal microbiota can be recovered with FMT, irrespective of its administration method. Safety remains a concern, ${ }^{107}$ especially in advanced GI aGvHD, and if an infectious complication occurs post-FMT, the pathogen should be sequenced and traced to find out if it originates from the FMT.

\section{Case 2: recommendations}

Currently, ruxolitinib is the only FDA-approved drug for the treatment of SR aGvHD, while other modalities are also commonly used in this scenario (e.g., extracorporeal photopheresis). Thus, FMT could be recommended for patients with grade 2-4 steroid refractory or dependent aGVHD of the lower GI tract, albeit in the context of a clinical study only. ${ }^{108-110}$ Other treatment approaches could also be considered, such as adding it to steroids as part of the first-line therapy (clinicaltrials gov. Identifier: 04269850).

Although clinical trials are still ongoing, given the grave prognosis of SR a GvHD with more than 50\% mortality, and the high rate of response to FMT, we recommend considering FMT as a therapeutic option in this setting.

\section{Practical considerations for fecal microbiota transplantation treatment}

As FMT has become the standard of care in recurrent and refractory $\mathrm{CDI},{ }^{112,113}$ more and more centers are gaining access to FMT programs through either establishing their own stool banks or acquiring FMT from universal stool banks. ${ }^{114,115}$

One of the limiting factors to wider application of stool banks and FMT programs is the lack or variance of regulatory standards. In different countries, FMT is regulated as a drug, tissue or a combined product composed of both human cells and non-human components (microbial DNA and metabolites). Stool banks are recommended to operate under the designated authority in each country. In the absence of local directives, the scientific committee should be responsible for establishing regulatory protocols..$^{14}$

FMT donor screening should follow national regulations and international recommendations. ${ }^{114}$ Screening should include medical history related to the risk for transmitting infections, as well as medical conditions and treatments associated with perturbed microbiome (Table 3). Special considerations are to be applied when planning FMT use in allo-HSCT patients, such as testing the donor for Cytomegalovirus and Epstein-Barr virus IgG and IgM, and administering FMT from seronegative donors to seronegative patients. However, when weighing suitability of an FMT donor, one should be cognizant of the fact that no data are available to support the advantage of a particular 
donor (a family member, an unrelated donor, or pooled stool from several unrelated donors).

As for autologous FMT, it has not been tested in the setting of aGvHD treatment. Since the microbiota composition of a patient is already disrupted prior to HSCT, using such stool in FMT preparation to be applied for diversity restoration may not be effective. In order to circumvent this problem, in AML patients, we recommend freezing self-stool before the beginning of induction chemotherapy.

In CDI, both fresh and frozen FMT have been shown to be efficient ${ }^{116}$ as have been the two delivery routes colonoscopy and oral capsules. ${ }^{117}$ While there are no data pointing to the superiority of either method of preparation or administration for aGvHD treatment, frozen samples from a stool bank allow FMT to be readily available for immediate use without the need to wait for donor screening and FMT collection.

The basic principles of FMT preparation include weighing the sample, suspension in sterile solution (saline), adding glycerol in case the FMT is planned for freezing and storing, homogenization, filtering and aliquoting the sus- pension for fresh use or freezing (Table 3). The FMT product should be registered and labeled. ${ }^{114}$

Based on the available data (Table 2) we suggest evaluating clinical response at 7-14 days after FMT administration. If no response or only partial response is achieved, we recommend administering a second dose of FMT. Whether in such cases the use of FMT from another donor could provide a superior outcome is yet to be determined. In general, in order to consider FMT as an efficacious therapeutic approach for SR GI aGvHD management, an overall response rate of around $60-70 \%$, with a complete response rate of $30-50 \%$ should be a desired target, as these rates are achieved with the use of the approved ruxolitinib treatment and in non-randomized FMT studies. ${ }^{46,98-106,110}$

As for the antibiotic treatment peri-FMT, if feasible, 2448 hours prior to FMT, systemic antibiotics should be stopped or replaced by one with less anti-anaerobic activity such as rifaximin for prophylaxis or cefepime for febrile neutropenic treatment. ${ }^{46,98,99}$

Microbiome sequencing of donor and patient samples could help interpreting clinical outcomes. It could also be

Table 3. Practical aspects of fecal microbiota transplantation.

FMT stool bank ${ }^{114}$

- Center's own bank

- Acquiring FMT from stool banks of other centers or from a universal stool bank

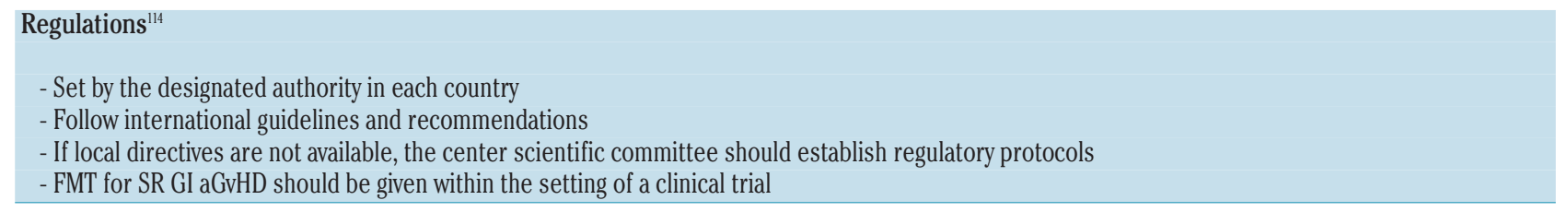

\section{FMT donor screening ${ }^{114 *}$}

Medical history for infections and risk for infections:

- HIV, hepatitis C, hepatitis B, syphilis, HTLV, other infections, malaria, tuberculosis, illegal drug use, unprotected sex, tissue/organ transplant, recent hospitalization, travel to high risk endemic countries, tattoo, piercing, earing, recent intestinal infection, recent vaccinations with live attenuated virus, blood transfusion, therapy with growth hormone.

\section{Medical history for conditions and medications with risk for microbiota perturbation:}

- Chronic gastrointestinal disease (e.g., inflammatory bowel disease, celiac disease), autoimmune disease, cancer, recent GI symptoms (e.g., diarrhea), neurologic disorders, psychiatric disorders, obesity, metabolic syndrome, diabetes, first degree relative with early colon cancer or polyposis. Antibiotic treatment in recent 3 months, chemotherapy, immunotherapy, prolonged use of proton-pump inhibitors, use of probiotics.

Blood tests:

- Hepatitis A, B and C, HTLV, HIV, treponema pallidum, strongyloides stercoralis, NAT for hepatitis B, C and HIV, ANCA (P and C), IgA antibodies level, anti-transglutaminase antibodies, antinuclear antibody, ASCA, liver enzymes, creatinine, calcium, albumin, cholesterol, triglycerides, complete blood count, thyroid function test.

Stool tests:

- Stool culture for Shigella, Salmonella and Campylobacter, direct smear for parasites from different occasions, Clostridium difficile antigen, vancomycin-resistant Enterococci (VRE), methicillin-resistant Staphylococcus aureus (MRSA), carbapenem-resistant Enterobacteriaceae (CRE), extended spectrum $\beta$ lactamase producing Enterobacteriaceae (ESBL), *Biofire (Biofire FilmArray) multiplex PCR for Yersinia enterocolitica, EAEC (Enteroaggregative E. coli), EPEC (Enteropathogenic E. coli), ETEC (Enterotoxigenic E. coli), STEC (Shiga-like toxin producing E. coli stx1/stx2), E. coli 0157, Shigella / EIEC (Enteroinvasive E. coli), Cryptosporidium, Cyclospora cayetanensis, Entamoeba histolytica, Giardia lamblia, Adenovirus F 40/41, Astrovirus, Norovirus, Rotavirus A, Sapovirus, Campylobacter, Clostridium difficile toxins A and B, Plesiomonas shigelloides, Salmonella, Vibrio parahaemolyticus and vulnificus, Vibrio cholerae.

\section{Special considerations:}

- Cytomegalovirus and Epstein-Barr virus serology ( $\operatorname{IgM}$ and $\operatorname{Ig} \mathrm{G}$ ) when administration to immunocompromised patients is planned. ${ }^{114}$

- Patients with severe food allergy should receive FMT from a donor who will avoid the allergy causing food for 72 hours prior to donation.*

- SARS-CoV-2 screening ${ }^{120}$ following the FDA safety alert. ${ }^{121}$

Consent:

- Both donors and patients should sign appropriate informed consent. 
FMT source ${ }^{122}$

-Fresh versus frozen: frozen is ready for immediate use.

- Unrelated donor, pooled stool from many unrelated donors, related donor, autologous collected while the patient was still healthy.

FMT preparation and storage ${ }^{144,122 *}$ (in brief)

- Donor stool collected into a sterile plastic container.

- If not done on site, it should be kept at $-4^{\circ} \mathrm{C}$ until processing.

- Stool processing and storage should be done within 6 hours from collection.

- Processing should be done in a sterile hood

- Weigh the stool (25 g minimum for lower GI FMT and $12.5 \mathrm{~g}$ for upper GI FMT).

- Mix with sterile saline, homogenize, filter and centrifuge.

- Re-suspend the pellet with saline.

- If stored frozen, add glycerol to a concentration of $10 \%$.

- Aliquot and label according to way of administration (capsules, tubes)

- Frozen FMT should be kept at $-80^{\circ} \mathrm{C}$ and preferably used within 1 year from collection.

\section{FMT administration ${ }^{11,122 *}$}

FMT preparation:

- Fresh FMT is given within 6-8 hours from collection.

- Frozen FMT is thawed at $37^{\circ} \mathrm{C}$ water bath and administered within 4-6 hours.

- Frozen capsules, thawed at room temperature for a few minutes.

\section{Method of administration:}

- Upper GI - gastroduodenoscopy, nasogastric tube, nasoduodenal tube, capsules.

- Lower GI - colonoscopy, enema.

Special considerations:

- If possible, to stop antibiotic treatment 24-48 hours prior to administration.

- Or replace current antibiotics with less anti-anaerobic antibiotics (e.g., rifaximin, cefepime)

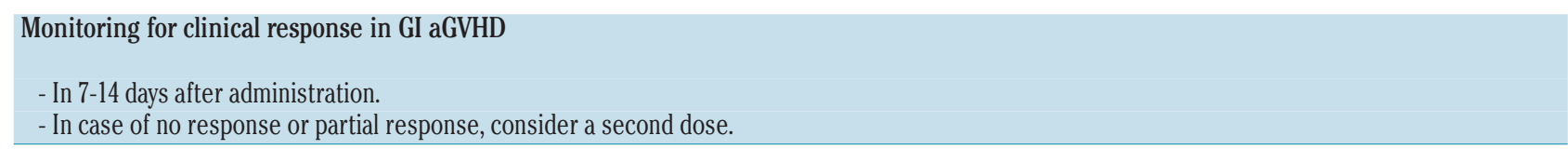

\section{Stool sampling for later sequencing (16S ribosomal RNA sequencing or other)}

\section{From donor:}

- A sample from the collected stool of each batch of donation.

\section{From patient:}

- A sample obtained before FMT, 1 week, 2 weeks and 4 weeks after FMT, at relapse/progression of GI aGvHD.

\section{Monitoring for adverse events ${ }^{122 *}$}

Commonly reported:

- Aspiration (in upper GI administration), nausea, vomiting, constipation, diarrhea, bloating, abdominal pain, adverse events caused by the nasogastric tube insertion or colonoscopy procedure, fever.

\section{Infections:}

- Diarrhea, colitis, bacteremia, pneumonia.

*National and Institutional guidelines.FMT: fecal microbiota transplantation,SR: steroid refractory, GI: gastrointestinal, aGvHD: acute graft-versus-host disease, HIV: human immunodeficiency virus, HTLV: human T-cell leukemia virus, NAT: nucleic acid test,ANCA: anti-neutrophil cytoplasmic antibodies,ASCA: anti- saccharomyces cerevisiae antibodies, CMV: cytomegalovirus, EBV: Epstein-Bar virus.

valuable in distinguishing between the donor and the recipient as the source of post-FMT infection. However, currently there are no data suggesting that patient stool sequencing prior to FMT could guide its administration or affect the outcome. Therefore, given that the primary outcome should be the clinical response to treatment we recommend treating SR GI aGvHD patients with FMT even if the microbiome analysis is not available. Nonetheless, we do suggest storing stool samples from the donor and the patient (before and after FMT) for later sequencing if it becomes available.

Further accumulation of data on FMT for SR GI aGvHD will allow wider and more efficient application of this treatment approach.

\section{Open challenges and future directions}

Disruption of the intestinal microbiome during alloHSCT is a multifaceted process with a cause-and-effect relationship between multiple factors such as conditioning, diet and antibiotic treatment. Lately, FMT has emerged as an intervention that can facilitate microbiome recovery and potentially intervene with the above interplay (Figure 1). The intestinal microbial disruption before and during allo-HSCT is clearly associated with transplant-related outcomes, mainly acute GvHD and mortality, and pre-clinical data demonstrate the key role of the intestinal microbiota in protecting the gut from inflammatory damage and in regulating the innate immune system to maintain a more tolerant state. ${ }^{118}$ 


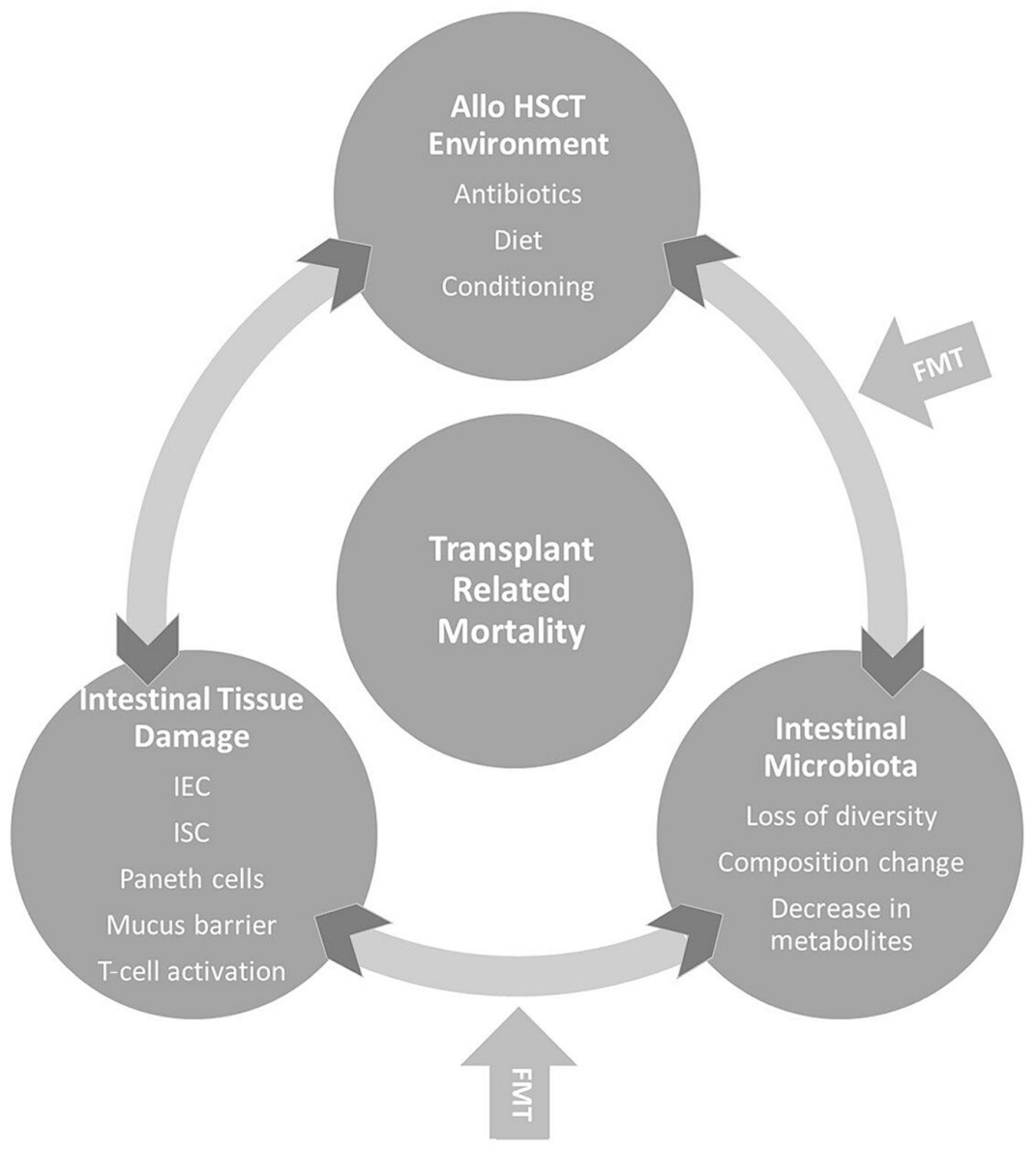

Figure 1. The multifactorial interplay between environmental factors, intestinal microbiota and tissue damage affects transplant-related outcomes. During allogeneic hematopoietic stem cell transplantation (allo-HSCT), conditioning chemotherapy causes damage to the intestinal mucosa cells such as intestinal epithelial cells, intestinal stem cells, paneth cells and mucus producing goblet cells. Gut microbiota is already disrupted before allo-HSCT and due to prophylactic and systemic antibiotic therapy the microbiota disruption worsens with loss of butyrate producing bacteria and other beneficial commensals, along with increase in pathogenic bacteria such as Enterococcus. Depletion of bacterial metabolites postpones epithelial cell repair and restoration of the mucus barrier. Pathogenic bacteria can disseminate through the damaged mucosa and cause blood stream infections, which will necessitate the administration of systemic antibiotics further disrupting the intestinal microbiota. This vicious cycle is associated with graft-versus-host disease (GvHD), increased mortality and diminished overall survival. The question remains whether fecal microbiota transplantation (FMT) and other interventions such as prebiotics and the use of antibiotics with less anti-anaerobic activity could eventually break the cycle and improve outcomes. IEC:intestinal epithelial cells; ISC: intestinal stem cells.

While the addition of beneficial bacteria or their metabolites has been shown to ameliorate acute GvHD in animal allo-HSCT models, many challenges remain concerning the role of the intestinal microbiota in allo-HSCT in humans. A substantial amount of basic research is being conducted aiming to better understand the place of microbiome changes in the pathogenesis of acute GvHD. In addition, a large population microbiome analysis is ongoing attempting to delineate the interplay between other factors, such as antibiotics and diet, and the microbiota disruption, and to determine the optimal strategy allowing to preserve the microbiota intact. ${ }^{119}$ However, while these issues are still under investigation, clinical trials evaluating the efficacy of FMT and other abovementioned interventions in the HSCT setting are under- way (Table 2). Joint efforts to further explore biological, correlative and recovery functions of the intestinal microbiota could ultimately lead to decreased transplantrelated mortality, and even pave the way to personalized therapeutic strategies in HSCT.

\section{Disclosures}

No conflicts of interest to disclose.

\section{Contributions}

$I H, D Y-O$ and $T Z$ wrote the paper.

\section{Acknowledgements}

The authors wish to thank Sonia Kamenetsky for her assistance in the preparation of this manuscript.

\section{References}

1. D'Souza A, Fretham C. Current uses and outcomes of hematopoietic cell transplantation (HCT): CIBMTR Summary Slides, 2019. Available at https://www.cibmtr.org. Accessed April, 2020. 2019.

2. Canaani J, Beohou E, Labopin M, et al. Trends in patient outcome over the past two decades following allogeneic stem cell transplantation for acute myeloid leukaemia: an ALWP/EBMT analysis. J Intern Med. 2019;285(4):407-418.
3. Turnbaugh PJ, Ley RE, Hamady M, FraserLiggett CM, Knight R, Gordon JI. The human microbiome project. Nature. 2007 449(7164):804-810.

4. Eckburg PB, Bik EM, Bernstein CN, et al. Diversity of the human intestinal microbia flora. Science. 2005;308(5728):1635-1638.

5. Candela M, Biagi E, Maccaferri S, Turroni S, Brigidi P. Intestinal microbiota is a plastic factor responding to environmental changes. Trends Microbiol. 2012;20(8):385-391.

6. Fulde M, Hornef MW. Maturation of the enteric mucosal innate immune system during the postnatal period. Immunol Rev.
2014;260(1):21-34.

7. Ijssennagger N, Belzer C, Hooiveld GJ, et al. Gut microbiota facilitates dietary hemeinduced epithelial hyperproliferation by opening the mucus barrier in colon. Proc Natl Acad Sci U S A. 2015;112(32):10038 10043.

8. Reinhardt C, Bergentall M, Greiner TU, et al. Tissue factor and PAR1 promote microbiotainduced intestinal vascular remodelling Nature. 2012;483(7391):627-631.

9. Neuman H, Debelius JW, Knight R, Koren O. Microbial endocrinology: the interplay between the microbiota and the endocrine 
system. FEMS Microbiol Rev. 2015;39(4): 509-521.

10. Canfora EE, Jocken JW, Blaak EE. Shortchain fatty acids in control of body weight and insulin sensitivity. Nat Rev Endocrinol. 2015;11(10):577-591.

11. Yatsunenko T, Rey FE, Manary MJ, et al. Human gut microbiome viewed across age and geography. Nature. 2012;486(7402):222227.

12. Devlin AS, Fischbach MA. A biosynthetic pathway for a prominent class of microbiota-derived bile acids. Nat Chem Biol. 2015;11(9):685-690.

13. Haiser HJ, Gootenberg DB, Chatman K, Sirasani G, Balskus EP, Turnbaugh PJ. Predicting and manipulating cardiac drug inactivation by the human gut bacterium Eggerthella lenta. Science. 2013;341(6143): 295-298.

14. Turnbaugh PJ, Hamady M, Yatsunenko T, et al. A core gut microbiome in obese and lean twins. Nature. 2009;457(7228):480-484.

15. Qin J, Li Y, Cai Z, et al. A metagenome-wide association study of gut microbiota in type 2 diabetes. Nature. 2012;490(7418):55-60.

16. Kostic AD, Xavier RJ, Gevers D. The microbiome in inflammatory bowel disease: current status and the future ahead. Gastroenterology. 2014;146(6):1489-1499.

17. Forbes JD, Van Domselaar G, Bernstein CN. The gut microbiota in immune-mediated inflammatory diseases. Front Microbiol. 2016;7:1081.

18. Poore GD, Kopylova E, Zhu Q, et al. Microbiome analyses of blood and tissues suggest cancer diagnostic approach. Nature. 2020;579(7800):567-574

19. Gopalakrishnan V, Spencer CN, Nezi L, et al. Gut microbiome modulates response to anti-PD-1 immunotherapy in melanoma patients. Science. 2018;359(6371):97-103

20. Zama D, Biagi E, Masetti R, et al. Gut microbiota and hematopoietic stem cell transplantation: where do we stand? Bone Marrow Transplant. 2017;52(1):7-14.

21. Hooper LV, Littman DR, Macpherson AJ. Interactions between the microbiota and the immune system. Science. 2012;336(6086): 1268-1273

22. Zhao Q, Elson CO. Adaptive immune education by gut microbiota antigens. Immunology. 2018;154(1):28-37.

23. Honda K, Littman DR. The microbiota in adaptive immune homeostasis and disease. Nature. 2016;535(7610):75-84.

24. Shouval R, Fein JA, Labopin M, et al. Outcomes of allogeneic haematopoietic stem cell transplantation from HLAmatched and alternative donors: a European Society for Blood and Marrow Transplantation registry retrospective analysis. Lancet Haematol. 2019;6(11):e573-e584.

25. DeFilipp Z, Peled JU, Li S, et al. Third-party fecal microbiota transplantation following allo-HCT reconstitutes microbiome diversity. Blood Adv. 2018;2(7):745-753

26. Montassier E, Batard E, Massart S, et al. 16S rRNA gene pyrosequencing reveals shift in patient faecal microbiota during high-dose chemotherapy as conditioning regimen for bone marrow transplantation. Microb Ecol. 2014:67(3):690-699

27. Bodet CA, 3rd, Jorgensen JH, Drutz DJ. Antibacterial activities of antineoplastic agents. Antimicrob Agents Chemother. 1985;28(3):437-439.

28. Hamilton-Miller JM. Antimicrobial activity of 21 anti-neoplastic agents. Br J Cancer. 1984;49(3):367-369.

29. Schirmer M, Smeekens SP, Vlamakis H, et al.
Linking the human gut microbiome to inflammatory cytokine production capacity. Cell. 2016;167(4):1125-1136.e8.

30. Swimm A, Giver CR, DeFilipp Z, et al. Indoles derived from intestinal microbiota act via type I interferon signaling to limit graft-versus-host disease. Blood. 2018;132 (23):2506-2519

31. Mathewson ND, Jenq R, Mathew AV, et al. Gut microbiome-derived metabolites modulate intestinal epithelial cell damage and mitigate graft-versus-host disease. Nat Immunol. 2016;17(5):505-513.

32. Sandhu SS, Pourang A, Sivamani RK. A review of next generation sequencing technologies used in the evaluation of the skin microbiome: what a time to be alive. Dermatol Online J. 2019;25(7):13030/ qt3hv5z3q3.

33. Marchesi JR, Ravel J. The vocabulary of microbiome research: a proposal. Microbiome. 2015;3:31

34. Rinninella E, Raoul P, Cintoni M, et al. What is the healthy gut microbiota composition? A Changing ecosystem across age, environment, diet, and diseases. Microorganisms. 2019;7(1):14.

35. Wortelboer K, Nieuwdorp M, Herrema H. Fecal microbiota transplantation beyond Clostridioides difficile infections. EBioMedicine. 2019:44:716-729.

36. Ooijevaar RE, Terveer EM, Verspaget HW Kuijper EJ, Keller JJ. Clinical application and potential of fecal microbiota transplantation. Annu Rev Med. 2019;70:335-351.

37. Peled JU, Gomes ALC, Devlin SM, et al. Microbiota as predictor of mortality in allogeneic hematopoietic-cell transplantation. $\mathrm{N}$ Engl J Med. 2020;382(9):822-834.

38. Mancini N, Greco R, Pasciuta R, et al. Enteric microbiome markers as early predictors of clinical outcome in allogeneic hematopoietic stem cell transplant: results of a prospective study in adult patients. Open Forum Infect Dis. 2017;4(4):ofx215

39. Staffas A, Burgos da Silva M, van den Brink MR. The intestinal microbiota in allogeneic hematopoietic cell transplant and graft-versus-host disease. Blood. 2017;129(8):927933.

40. Taur Y, Jenq RR, Perales MA, et al. The effects of intestinal tract bacterial diversity on mortality following allogeneic hematopoietic stem cell transplantation. Blood. 2014:124(7):1174-1182.

41. Lee YJ, Arguello ES, Jenq RR, et al. Protective factors in the intestinal microbiome against clostridium difficile infection in recipients of allogeneic hematopoietic stem cell transplantation. J Infect Dis. 2017;215(7):1117-1123

42. Andermann TM, Peled JU, Ho C, et al. The Microbiome and hematopoietic cell transplantation: past, present, and future. Biol Blood Marrow Transplant. 2018;24(7):13221340

43. Ladas EJ, Bhatia M, Chen L, et al. The safety and feasibility of probiotics in children and adolescents undergoing hematopoietic cell transplantation. Bone Marrow Transplant. 2016:51(2):262-266

44. Iyama S, Sato T, Tatsumi H, et al. Efficacy of enteral supplementation enriched with glutamine, fiber, and oligosaccharide on mucosal injury following hematopoietic stem cell transplantation. Case Rep Oncol. 2014;7(3): 692-699.

45. Weber D, Oefner PJ, Dettmer K, et al. Rifaximin preserves intestinal microbiota balance in patients undergoing allogeneic stem cell transplantation. Bone Marrow Transplant. 2016;51(8):1087-1092.
46. Kakihana K, Fujioka Y, Suda W, et al. Fecal microbiota transplantation for patients with steroid-resistant acute graft-versus-host disease of the gut. Blood. 2016;128(16):20832088

47. Galloway-Pena JR, Smith DP Sahasrabhojane P, et al. The role of the gastrointestinal microbiome in infectious complications during induction chemotherapy for acute myeloid leukemia. Cancer. 2016;122(14):2186-2196.

48. Rashidi A, Kaiser T, Shields-Cutler R, et al. Dysbiosis patterns during re-induction/salvage versus induction chemotherapy for acute leukemia. Sci Rep. 2019;9(1):6083.

49. Kusakabe S, Fukushima K, Maeda T, et al. Pre- and post-serial metagenomic analysis of gut microbiota as a prognostic factor in patients undergoing haematopoietic stem cell transplantation. Br J Haematol. 2020;188 (3):438-449

50. Taur Y, Xavier JB, Lipuma L, et al. Intestinal domination and the risk of bacteremia in patients undergoing allogeneic hematopoietic stem cell transplantation. Clin Infect Dis. 2012;55(7):905-914

51. Biagi E, Zama D, Nastasi C, et al. Gut microbiota trajectory in pediatric patients undergoing hematopoietic SCT. Bone Marrow Transplant. 2015;50(7):992-998.

52. Holler E, Butzhammer P, Schmid K, et al. Metagenomic analysis of the stool microbiome in patients receiving allogeneic stem cell transplantation: loss of diversity is associated with use of systemic antibiotics and more pronounced in gastrointestinal graft-versushost disease. Biol Blood Marrow Transplant. 2014;20(5):640-645

53. Taur Y, Coyte K, Schluter J, et al. Reconstitution of the gut microbiota of antibiotic-treated patients by autologous fecal microbiota transplant. Sci Transl Med. 2018;10(460):eaap9489.

54. Kusakabe S, Fukushima K, Yokota T, et al. Enterococcus: A Predictor of ravaged microbiota and poor prognosis after allogeneic hematopoietic stem cell transplantation. Biol Blood Marrow Transplant. 2020;26(5):10281033.

55. Liu C, Frank DN, Horch $M$, et al Associations between acute gastrointestinal GvHD and the baseline gut microbiota of allogeneic hematopoietic stem cell transplant recipients and donors. Bone Marrow Transplant. 2017;52(12):1643-1650.

56. Golob JL, Pergam SA, Srinivasan S, et al Stool microbiota at neutrophil recovery is predictive for severe acute graft vs host disease after hematopoietic cell transplantation. Clin Infect Dis. 2017;65(12):1984-1991

57. Jenq RR, Taur Y, Devlin SM, et al. Intestinal Blautia is associated with reduced death from graft-versus-host disease. Biol Blood Marrow Transplant. 2015;21(8):1373-1383.

58. Biagi E, Zama D, Rampelli S, et al. Early gut microbiota signature of aGvHD in children given allogeneic hematopoietic cell transplantation for hematological disorders. BMC Med Genomics. 2019;12(1):49.

59. Peled JU, Devlin SM, Staffas A, et al. Intestinal microbiota and relapse after hematopoietic-cell transplantation. J Clin Oncol. 2017:35(15):1650-1659.

60. Forcina A, Lorentino F, Marasco V, et al Clinical impact of pretransplant multidrugresistant gram-negative colonization in autologous and allogeneic hematopoietic stem cell transplantation. Biol Blood Marrow Transplant. 2018;24(7):1476-1482

61. Sadowska-Klasa A, Piekarska A, Prejzner W, Bieniaszewska M, Hellmann A 
Fecal microbial transplant in HSCT

Colonization with multidrug-resistant actenia increases the risk of complications and a fatal outcome after allogeneic hematopoietc cell transplantation. Ann Hematol. 2018;97(3):509-517.

62. Bilinski J, Robak K, Peric Z, et al. Impact of gut colonization by antibiotic-resistant facterra on the outcomes of allogeneic hematopoietic stem cell transplantation: a retrospective, single-center study. Biol Blood Marrow Transplant. 2016;22(6):1087-1093.

63. Oren I, Sprecher H, Finkelstein R, et al. Eradication of carbapenem-resistant Enterobacteriaceae gastrointestinal colonization with nonabsorbable oral antibiotic treatment: a prospective controlled trial. Am J Infect Control. 2013;41(12):1167-1172.

64. Battipaglia G, Malard F, Rubio MT, et al. Fecal microbiota transplantation before or after allogeneic hematopoietic transplantaton in patients with hematologic malignancues carrying multidrug-resistance bacteria. Haematological. 2019;104(8):1682-1688.

65. Ines AJ, Mulish BH, Fernando F, et al. Faecal microbiota transplant: a novel biologicel approach to extensively drug-resistant organism-related non-relapse mortality. Bone Marrow Transplant. 2017;52(10):14521454.

66. Malard F, Vekhoff A, Lapusan S, et al. The ODYSSEE study: Prevention of dysbiosis complications with tautologous fecal microbiota transfer in acute myeloid leukemia patients undergoing intensive-treatment: Results of a prospective multicenter trial. Bone Marrow Transplant. 2019;54:OS16-11

67. Willems L, Porcher R, Lafaurie $M$, et al. Clostridium difficile infection after allogeneic hematopoietic stem cell transplantation: incidene, risk factors, and outcome. Biol Blood Marrow Transplant. 2012;18(8):1295-1301.

68. Alonso CD, Treadway SB, Hanna DB, et al. Epidemiology and outcomes of Clostridium difficile infections in hematopoietic stem cell transplant recipients. Chin Infect Dis. 2012;54(8):1053-1063.

69. Dubberke ER, Reske KA, Olsen MA, et al. Epidemiology and outcomes of Clostridium difficile infection in allogeneic hematopoietic cell and lung transplant recipients. Transpl Infect Dis. 2018;20(2):e12855.

70. Kinnebrew MA, Lee YJ, Jenq RR, et al. Early Clostridium difficile infection during allgenic hematopoietic stem cell transplantatimon. PLo One. 2014;9(3):e90158.

71. Webb BJ, Brynner A, Ford CD, Gazdik MA, Petersen FB, Hoda D. Fecal microbiota transplantation for recurrent Clostridium difficile infection in hematopoietic stem cell transplant recipients. Transpl Infect Dis. 2016;18(4):628-633.

72. Moss EL, Falconer SB, Tkachenko E, et al. Long-term taxonomic and functional diverfence from donor bacterial strains following fecal microbiota transplantation in immunocompromised patients. CLoS One. 2017;12(8):e0182585.

73. Bluestone H, Kronman MP, Suskind DL. Fecal microbiota transplantation for recurrent Clostridium difficile infections in periatric hematopoietic stem cell transplant recipients. J Pediatric Infect Dis Soc. 2018;7(1):e6-e8.

74. Han $\mathrm{L}$, Chang $\mathrm{H}$, Chen $\mathrm{S}$, et al. Intestinal microbiota can predict acute graft-versushost disease following allogeneic hematopoietic stem cell transplantation. Biol Blood Marrow Transplant. 2019;25(10): 1944-1955.

75. Kroger N, Iacobelli S, Franke GN, et al. Dose-reduced versus standard conditioning followed by allogeneic stem-cell transplantation for patients with myelodysplastic syndrome: A Prospective randomized phase III study of the EBMT (RICMAC Trial). J Chin Oncol. 2017;35(19):2157-2164.

76. Scott BL. Long-Term Follow up of BMT CTN 0901, a randomized phase III trial comparing myeloablative (MAC) to reduced intensity conditioning (RIC) prior to hematopoietic cell transplantation (HCT) for acute myeloid leukemia (AML) or myelodysplasia (MDS) (MAvRIC Trial). Biol Blood Marrow Transplant. 2020;26(3):S11.

77. Sengsayadeth S, Gatwood KS, Boumendil A, et al. Conditioning intensity in secondary AML with prior myelodysplastic syndrome/myeloproliferative disorders: an EBMT ALWP study. Blood Adv. 2018;2(16): 2127-2135.

78. D'Amico F, Bragi E, Rampelli S, et al. Enteral nutrition in pediatric patients undergoing hematopoietic SCT promotes the recovery of gut microbiome homeostasis. Nutrients. 2019;11(12):2958.

79. Venkataraman A, Sieber JR, Schmidt AW, Waldron C, Their KR, Schmidt TM. Variable responses of human microbiomes to dietary supplementation with resistant starch. Microbiome. 2016;4(1):33.

80. Rimes MM, Schmidt A, Braun T, et al. Rational modification of intestinal microbicme and metabolites after allogeneic hematopoietic stem cell transplantation with resistant starch: a pilot study. Blood. 2019;134(Suppl_1):3276.

81. Gorshein E, Wei C, Ambrosy S, et al. Lactobacillus rhamnosus GG probiotic enteric regimen does not appreciably alter the gut microbiome or provide protection against GVHD after allogeneic hematopoietic stem cell transplantation. Chin Transplant. 2017;31(5).

82. Boyle RJ, Robins-Browne RM, Tang ML. Probiotic use in clinical practice: what are the risks? Am J Chin Nutr. 2006;83(6):12561264; quiz 1446-1257.

83. Stein-Thoeringer CK, Nichols KB, Lazrak A, et al. Lactose drives Enterococcus expansion to promote graft-versus-host disease. Science. 2019;366(6469):1143-1149.

84. Khandelwal P, Andersen H, RomickRosendale L, et al. A pilot study of human milk to reduce intestinal inflammation after bone marrow transplant. Breastfeed Med. 2019;14(3):193-202.

85. Gafter-Gvili A, Fraser A, Paul M, et al. Antibiotic prophylaxis for bacterial infectons in afebrile neutropenic patients following chemotherapy. Cochrane Database Syst Rev. 2012;1:CD004386.

86. Tomblyn M, Brunstein C, Burns LJ, et al. Similar and promising outcomes in lemphoma patients treated with myeloablative or nonmyeloablative conditioning and allogeneric hematopoietic cell transplantation. Biol Blood Marrow Transplant. 2008;14(5): 538-545.

87. Averbuch D, Orasch C, Cordonnier C, et al. European guidelines for empirical antibactrial therapy for febrile neutropenic patients in the era of growing resistance: summary of the 2011 th European Conference on Infections in Leukemia. Haematological. 2013;98(12):1826-1835.

88. Weber D, Oefner PJ, Hiergeist A, et al. Low urinary indoxyl sulfate levels early after transplantation reflect a disrupted microbicme and are associated with poor outcome. Blood. 2015;126(14):1723-1728.

89. Weber D, Hiergeist A, Weber $M$, et al. Detrimental Effect of broad-spectrum

antibiotics on intestinal microbiome diversity in patients after allogeneic stem cell transplantation: lack of commensal sparing antibiotics. Chin Infect Dis. 2019;68(8):13031310.

90. Pouty B, Letendre C, Enot D, et al. The influence of gut-decontamination trophylactic antibiotics on acute graft-versus-host disease and survival following allogeneic hematopoietic stem cell transplantation. Oncoimmunology. 2017;6(1):e1258506.

91. Weber D, Jena RR, Pelee JU, et al. Microbiota disruption induced by early use of broad-spectrum antibiotics is an ingependent risk factor of outcome after altogenic stem cell transplantation. Biol Blood Marrow Transplant. 2017;23(5):845-852.

92. Morjaria S, Schluter J, Taylor BP, et al. Antibiotic-induced shifts in fecal microbiota density and composition during hematopoitic stem cell transplantation. Infect Immun. 2019;87(9):e00206-e00219.

93. Simms-Waldrip TR, Sunkersett G, Coughlin LA, et al. Antibiotic-induced depletion of anti-inflammatory Clostridia is associated with the development of graft-versus-host disease in pediatric stem cell transplantation patients. Biol Blood Marrow Transplant. 2017;23(5):820-829.

94. Lee SE, Dim JY, Ry DB, et al. Alteration of the intestinal microbiota by broad-spectrum antibiotic use correlates with the occurrence of intestinal graft-versus-host disease. Biol Blood Marrow Transplant. 2019;25(10): 1933-1943.

95. Shone Y, Docampo MD, Paled JU, et al. Increased GVHD-related mortality with broad-spectrum antibiotic use after allogenic hematopoietic stem cell transplantstron in human patients and mice. Sci Transl Med. 2016;8(339):339ra371.

96. Romick-Rosendale LE, Haslam DB, Lane A, et al. Antibiotic exposure and reduced short chain fatty acid production after hematopoitic stem cell transplant. Biol Blood Marrow Transplant. 2018;24(12):2418-2424.

97. Nishi K, Kanda J, Hishizawa M, et al. Impact of the Use and type of antibiotics on acute graft-versus-host disease. Biol Blood Marrow Transplant. 2018;24(11):2178-2183.

98. Spindelboeck W, Schulz E, Ul B, et al. Repeated fecal microbiota transplantation attenuate diarrhea and lead to sustained changes in the fecal microbiota in acute, refractory gastrointestinal graft-versus-hostdisease. Haematological. 2017;102(5):e210e213.

99. Kaito S, Toy T, Yoshifuji K, et al. Fecal microbiota transplantation with frozen capsoles for a patient with refractory acute gut graft-versus-host disease. Blood Adv. 2018;2(22):3097-3101.

100. Chang J, Ken G, Li M, Lu P, Ye S. The effects of fecal donors with different feeding patterns on diarrhea in a patient undergoing hematopoietic stem cell transplantation. Case Rep Hematol. 2019;2019:4505238.

101. Zhong S, Zens J, Deng Z, et al. Fecal microbiota transplantation for refractory diarrhea in immunocompromised diseases: a periatric case report. Ital J Pediatr. 2019;45 (1):116.

102. Shouval R, Youngster I, Geva M, et al. Repeated courses of orally administered fecal microbiota transplantation for the treatment of steroid resistant and steroid dependent intestinal acute graft vs. host disease: A pilot study (NCT 03214289). Blood. 2018;132(Suppl_1):2121.

103. Malard F, Legrand F, Cornillon J, et al. Successful and safe treatment of intestinal 
graft-versus-host disease (GvHD) with pooled-donor full ecosystem microbiota biotherapeutics. Blood. 2019;134(Suppl_1): 1993.

104. Oi X, Li X, Zhao Y, et al. Treating steroid refractory intestinal acute graft-vs.-host disease with fecal microbiota transplantation: A pilot study. Front Immunol. 2018;9:2195.

105. van Lier YF, M D, Haverkate NJE, et al. Fecal microbiota transplantation can cure steroidrefractory intestinal graft-versus-host disease. Biol Blood Marrow Transplant 2019;25(3):S241.

106. Bilinski J, Lis K, Tomaszewska A, et al. Fecal microbiota transplantation as a treatment of severe steroid-resistant acute and chronic graft versus host disease. Spectrum of responses and complications. Blood. 2019;134(Suppl_1):5667.

107. DeFilipp Z, Bloom PP, Torres Soto M, et al. Drug-resistant E. coli bacteremia transmitted by fecal microbiota transplant. N Engl J Med. 2019;381(21):2043-2050.

108. Modemann F, Ayuk F, Wolschke C, et al. Ruxolitinib plus extracorporeal photopheresis (ECP) for steroid refractory acute graft-versus-host disease of lower GI-tract after allogeneic stem cell transplantation leads to increased regulatory $\mathrm{T}$ cell level. Bone Marrow Transplant. 2020;55(12): 2286-2293.

109. Drexler B, Buser A, Infanti L, Stehle G, Halter J, Holbro A. Extracorporeal photopheresis in graft-versus-host disease. Transfus Med Hemother. 2020;47(3):214-225.

110. Zeiser R, von Bubnoff N, Butler J, et al.
Ruxolitinib for glucocorticoid-refractory acute graft-versus-host disease. N Engl J Med. 2020;382(19):1800-1810.

111. Srinagesh HK, Levine JE, Ferrara JLM. Biomarkers in acute graft-versus-host disease: new insights. Ther Adv Hematol. 2019:10:2040620719891358.

112. Bakken JS, Borody T, Brandt LJ, et al Treating Clostridium difficile infection with fecal microbiota transplantation. Clin Gastroenterol Hepatol. 2011;9(12):10441049.

113. Davidovics ZH, Michail S, Nicholson MR, et al. Fecal microbiota transplantation for recurrent clostridium difficile infection and other conditions in children: A joint position paper from the North American Society for Pediatric Gastroenterology, Hepatology, and Nutrition and the European Society for Pediatric Gastroenterology, Hepatology, and Nutrition. J Pediatr Gastroenterol Nutr. 2019;68(1):130-143

114. Cammarota G, Ianiro G, Kelly CR, et al International consensus conference on stool banking for faecal microbiota transplantation in clinical practice. Gut. 2019;68(12): 2111-2121.

115. Panchal P, Budree S, Scheeler A, et al. Scaling safe access to fecal microbiota transplantation: past, present, and future. Curr Gastroenterol Rep. 2018;20(4):14

116. Lee CH, Steiner T, Petrof EO, et al. Frozen vs fresh fecal microbiota transplantation and clinical resolution of diarrhea in patients with recurrent Clostridium difficile infec- tion: a randomized clinical trial. JAMA. 2016;315(2):142-149.

117. Kao D, Roach B, Silva M, et al. Effect of oral capsule- vs colonoscopy-delivered fecal microbiota transplantation on recurrent Clostridium difficile infection: a randomized clinical trial. JAMA. 2017;318(20):1985-1993.

118. Riwes M, Reddy P. Microbial metabolites and graft versus host disease. Am J Transplant. 2018;18(1):23-29.

119. Nguyen CL, Gomes ALC, Peled JU, et al Antibiotic exposures and dietary intakes are associated with changes in microbiota compositions in allogeneic hematopoietic stem cell transplant patients. Blood. 2019;134 (Suppl_1):597.

120. Ng SC, Chan FKL, Chan PKS. Screening FMT donors during the COVID-19 pandemic: a protocol for stool SARS-CoV-2 viral quantification. Lancet Gastroenterol Hepatol. 2020;5(7):642-643.

121. Safety alert regarding use of fecal microbiota for transplantation and additional safety protections pertaining to SARS-CoV-2 and COVID-19. Available https://www.fda.gov/vaccines-blood-biologics/safety-availability-biologics/safetyalert-regarding-use-fecal-microbiota-transplantation-and-additional-safety-protections. Accessed on July 20, 2020.

122. Nicco C, Paule A, Konturek P, Edeas M From donor to patient: Collection, preparation and cryopreservation of fecal samples for fecal microbiota transplantation. Diseases. 2020;8(2):9. 